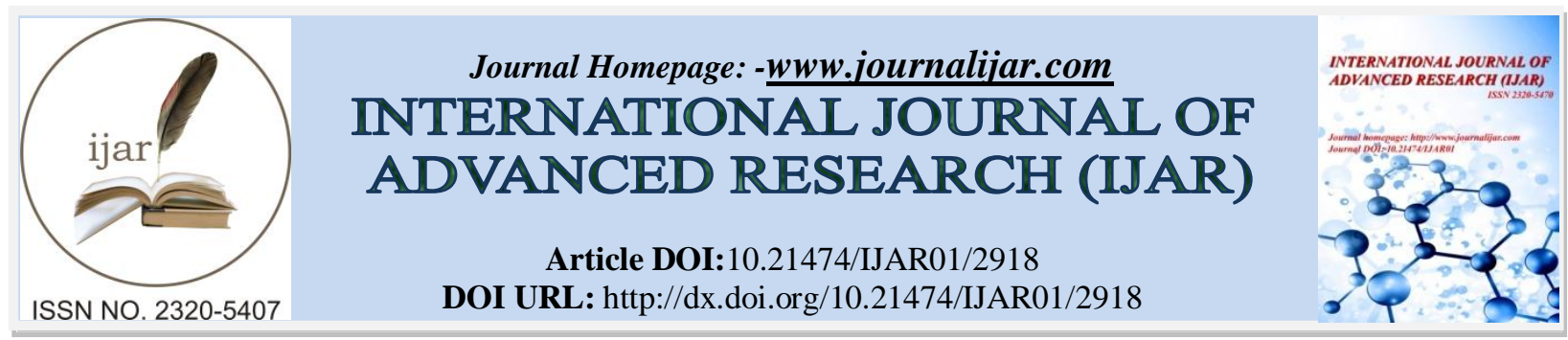

RESEARCH ARTICLE

\title{
METABOLIC SYNDROME AND ELEVATEDOSTEOPONTIN: THEIR ASSOCIATED COMORBIDITIES IN PATIENTS WITH PSORIASIS.
}

Hoda G. Bakr ${ }^{1}$, Fatma Eldesouky MDS ${ }^{2}$, Abdallah $\mathrm{H}_{\text {kandil }} \mathrm{MD}^{2}$ and Samar M. Sharaf MD ${ }^{3}$.

1. Department of Internal Medicine. Faculty of Medicine, Zagazig University, Egypt.

2. Department of Dermatology.Faculty of Medicine, Zagazig University, Egypt.

3. Department of Clinical pathology' Faculty of Medicine, Zagazig University, Egypt.

\section{Manuscript Info}

\section{Manuscript History}

Received: 24 November 2016

Final Accepted: 25 December 2016

Published: January 2017

Key words:-

Metabolic syndrome, Osteopontin

,Psoriasis

\section{Abstract}

Background:Psoriasis is a systemic, chronic, and hyper-proliferative immune mediated skin disorder.Osteopontin influences cell mediated immunity and plays an important role during both acute and chronic inflammation.

We aimed to assess metabolic syndrome and osteopontin and their associated systemic comorbidities in patients with psoriasis.

Subjects and methods: The study included 48 subjects: 24 patients of psoriasis of any age, both sexes with different grades of disease severity. Severity was assessed by psoriasis area and severity index (PASI) score, .Their mean age was $(33.25 \pm 12.9), 13$ of them were females $(54.2 \%)$ and 11 were males $(45.8 \%)$. In addition to 24 healthy control group, 15 females $(62.5 \%)$ and 9 males $(37.5 \%)$. Their mean age was $(31.63 \pm 16.52)$. Clinical, demographic evaluation and dermatological examination of the psoriatic lesions (site of the lesion, morphology and progression of the disease and severity ) was performed in addition to serological testing of serum OPN level, total cholesterol, low-density lipoprotein cholesterol, high-density lipoprotein cholesterol, triglyceride, and fasting blood glucose(FBG).

Results:psoriatic cases showed,elevatedbody mass index,FBG, serum osteopontin, cholesterol, triglycerides and low density lipoprotein cholesterol and decreased levels of high density lipoprotein cholesterol than normal control.Metabolic syndrome and hypertension representing $25 \%$ and $41.6 \%$ respectively of psoriatic cases.In psoriatic cases, psoriasis area and severity index (PASI) score, had significant positive correlation with obesity,blood pressure, FBG, dyslipidemia,but no correlation with age, disease duration.Serum OPN had positive correlation with age,blood pressure but no correlation with BMI, disease duration, FBS ordyslipidemia.

Conclusions Psoriatic patients have a high prevalence of metabolic syndrome. High level of OPN can be used as an early detector of cardiovascular problems in psoriatic patients and this inflammatory cytokine can be used as a new target in the treatment of psoriasis. 


\section{Introduction:-}

Psoriasis (PS) is a systemic, chronic, relapsing and hyper-proliferative immune mediated skin disorder. It affects 1-3 \% of the world population and its prevalence is highly variable, depending on ethnicity and climate. It is characterized by inflammatory skin and joint manifestations [1].

Psoriasis results from interaction between genetic predisposition and large spectrum of environmental factors that triggers the development of skin lesion. T-helper (Th) 1 and Th17 mediated inflammation have been proposed to be pathologically essentialin psoriasis [2].

Metabolic syndrome (Met.S) is a clinical entity comprising risk factors such as hypertension, glucose intolerance, hyperlipidemia, abdominal obesity and increased inflammatory state. Psoriasis patients may similarly predispose for those inflammatory disorders. Experimental and epidemiological studies have linked certain interleukins, cytokines and hormones with cardiovascular disease(CVD). Metabolic syndrome (Met.S), obesity and DM, making psoriasis at risk in developing systemic comorbidities[3].

Osteopontin (OPN) is phosphorylated sialic acid -rich non-collagenous bone matrix protein. OPN is found in several biological fluids including human plasma, serum, breast milk and urine.It was named for its function as a bridge between cells and minerals. OPN has been implicated as an important factor in bone remodeling and expressed in immune cells, including macrophages, neutrophils, dendritic cells with varying kinetics[4].Osteopontin influences cell mediated immunity and has Th 1 cytokine functions and is overexpressed in cancers of lung, breast, colorectal, stomach and ovary and found in atheromatous plaques within arteries. OPN plays an important role during both acute and chronic inflammation and is upregulated in tissues during several pathological process including atherosclerosis, valve stenosis, myocardial infarction and rheumatic arthritis[5].

Psoriasis is associated with cardiovascular comorbidities, possibly due to overall systemic inflammation. There is increased risk for atrial fibrillation, atherosclerosis,coronary artery calcification, type 2 diabetes,dyslipidemia, hypertension, obesity, peripheral vascular disease, myocardial infarction, stroke, and cardiac death. Also systemic treatments for psoriasis include cyclosporine, methotrexate, retinoids, and biological drugs have adverse cardio vascular (CV) effects. Inflammation may be a prime factor in linking psoriasis to atherosclerosis. The two conditions share many similarities, both psoriasis and atherosclerosis are mainly mediated by T-helper (Th) 1 cells and characterized by a systemic overexpression of adhesion molecules, inflammatory markers, and neo-angiogenesis factors.[6].

Osteopontin promoting the migration and activation of human DC and enhanced proliferation of vascular smooth muscle cells.Osteopontin expression increases under a variety of conditions of heart diseases and is associated with increased myocytic apoptosis and myocardial dysfunction[7].

Plasma OPN levels are higher in patients with coronary artery disease and have been correlated with its severity.Furthermore, plasma OPN has been reported to be a potential clinical marker for the prediction of atherosclerosis and further adverse cardiac events in patients with essential hypertension and patients with chronic stable angina. Osteopontin has also been implicated in the process of pathological vascular calcification. Increased osteopontin expression has been demonstrated in calcified human atherosclerotic plaques[8].

We aimed to assess metabolic syndrome and osteopontin and their associated systemic comorbidities in patients with psoriasis

\section{Patients and Methods:-}

This study was carried out at the outpatient clinics of Dermatology,Veneroloy and Andrology and Internal Medicine Departments, Faculty of medicine of Zagazig University,in the period from march 2015 till march 2016. The study had the approval of The Institutional Review Board (IRB) at Zagazig University, Egypt.

\section{Subjects:-}

The study included 48 subjects:24 patients ofnewly diagnosed psoriasis of any age, both sexes with different grades of disease severity, different clinical presentations.Severity was assessed by Psoriasis area and severity index (PASI) score, 13 of them were females $(54.2 \%)$ and 11 were males $(45.8 \%)$. Their ages ranged 10-60 year with a mean 
(33.25 \pm 12.9$)$. In addition to 24 control group, 15 females (62.5\%) and 9 males (37.5\%). Their ages ranged 10-70 with mean $(31.63 \pm 16.52)$.

\section{Exclusion criteria:-}

Patients receiving any systemic treatment for psoriasis for at least 2 months before inclusion in the study.Also we excluded alcohol consumers, smokers, patients on lipid lowering medications, corticosteroids or immunosuppressive drugs, respiratory, hepatic or renal diseasesand also patients with thyroid dysfunction.

\section{Methods:-}

They consented to be included in the study. In addition to full history, physical examination was performed with stress on weight, height and body mass index (BMI)which was calculated by dividing weight (kg) by height in squared meters $\left(\mathrm{m}^{2}\right)$. Dermatological examination of the psoriatic lesions (site of the lesion, morphology and progression of the disease and severity ).

Assessment of disease severity: According to Matteo and Michela, (2013) [9]: Psoriasis area and severity index (PASI) score is a three point disease activity score that has been used to grade the disease severity. The score assesses four body regions : the head $(\mathrm{H})$,the upper extremities $(\mathrm{U})$, the trunk $(\mathrm{T})$ and the lower extremities $(\mathrm{L})$ corresponding to $10 \%, 20 \%, 30 \%$ and $40 \%$ of the total body surface area respectively. According to the extent of the lesion, each area of psoriasis involvement is given a numerical value $(0$ - 6)corresponding to the following scale. The PASI result is a score for psoriasis severity ranges from 0 (no disease) to 72(maximal disease)according to Langley and Ellis, 2004[10].

\section{Procedure:-}

\section{Serological evaluation:-}

Venous blood samples $(5 \mathrm{ml})$ were collected under sterile conditions from all subjects to estimate serum OPN level by enzyme linked immunesorbent assay (ELISA) technique using a kit based on the principle of double-antibody sandwich technique and also to estimate lipid profile and FBS.

\section{Sample Collection and Storage:-}

Using a serum separator tube (SST), five $\mathrm{ml}$ of the blood sample was left to clot for 20 minutes at room temperature then subjected to centrifugation for 20 minutes at approximately 2000-3000 rpm to separate the serum. The serum samples were removed using a sterile pipette and stored at $-70^{\circ} \mathrm{C}$ until assayed.

\section{Test principle:-}

The kit used a double-antibody sandwich enzyme -linked immunosorbent assay (ELISA) to assay the level of human osteopontin (OPN) in samples. We add osteopontin (OPN) to monoclonal antibody enzyme which is precoated with human (OPN) monoclonal antibody,incubation,then we add (OPN) antibodies labeled with biotin and combined with streptavidin-HRP to form immune complex, then carry out incubation and washing again to remove the uncombined enzyme. Then we add chromogen solution A,B, the colour of the liquid changes into the blue and at the effect of acid ,the color finally becomes yellow. The chroma of the color and the concentration of the human substance (OPN) of sample were positively correlated.

The specific kit reagents used in the test were supplied from PELOBIOTECH GmbH - Am Klopferspitz 19 82152 Planegg - Germany.

\section{Statistical analysis:-}

The data were tabulated and statistically analyzed using Microsoft Office Excel 2010,and Statistical Package for Social Sciences version 20 (SPSS: An IBM Company). Data were represented as Mean \pm SD, and were analyzed statistically by using analysis of variance(ANOVA), Chi-squared test(X2), paired $\mathrm{T}$ test and correlation coefficient $(r)$.Values were considered significant if $\mathrm{p}<0.05$.

\section{Results:-}

Demographic and laboratory characteristics of patients and control groups are described in Table (1):We found no statistical difference in age and sex distributionbetween groups.But there was significantly elevated body mass index(BMI) inpsoriatic cases.Mean duration of disease was $7.92 \pm 8.06$, meanpsoriasis area and severity index 
(PASI) scorewas16.31 12.09 . Metabolic syndrome was present in $25 \%$ of psoriatic cases compared to $12.5 \%$ ofcontrol group, hypertension was present in $41.6 \%$ of psoriatic cases compared to $20.8 \%$ of control group but the difference was insignificant.

Table (2): The level of FBG in cases was higher than that of control ,but without statistically significant difference. There was significantly elevated levels of osteopontin, cholesterol, TG and LDL and significantly decreased levels of HDL in patients with psoriasis thancontrol .

Table (3), Fig. 1:In the psoriasis group, PASI score had no significant correlation with age or disease duration, butPASI score had significant +ve correlation withobesity(BMI), systolic \& diastolicblood pressure,FBG, dyslipidemia, (elevated cholesterol\& TG\& LDL) and significant -ve correlation between PASI score and HDL. InTable (3), Fig. 2:in the psoriasis group, serum OPN had significant +ve correlation with age,systolic and diastolic blood pressure. Serum OPN had no significant correlation with BMI, disease duration, FBG ,cholesterol,TG,LDL and HDL .

Table 1:- Demographic and clinical data of the studied subjects

\begin{tabular}{|c|c|c|c|c|}
\hline Variable & $\begin{array}{l}\text { Cases } \\
(n=24)\end{array}$ & $\begin{array}{c}\text { Control } \\
(\mathbf{n}=24)\end{array}$ & Test & $\mathbf{P}$ \\
\hline $\begin{array}{l}\text { Sex: n (\%) Female } \\
\text { Male }\end{array}$ & $\begin{array}{l}13(54.2 \%) \\
11(45.8 \%)\end{array}$ & $\begin{array}{l}15(62.5 \%) \\
9(37.5 \%)\end{array}$ & $\mathrm{X}^{2}=0.34$ & 0.56 \\
\hline Age (year) & $\begin{array}{c}33.25 \pm 12.9 \\
10-60\end{array}$ & $\begin{array}{c}31.63 \pm 16.52 \\
(10-70)\end{array}$ & $\mathrm{T}=0.6$ & 0.55 \\
\hline Duration of disease: (year) & $\begin{array}{r}7.92 \pm 8.06 \\
(0.17-33) \\
\end{array}$ & - & - & - \\
\hline $\begin{array}{l}\text { Psoriasis area and severity index } \\
\text { (PASI) score }\end{array}$ & $\begin{array}{c}16.31 \pm 12.09 \\
(1.8-41.5)\end{array}$ & - & - & - \\
\hline $\mathrm{BMI} \mathrm{kg} / \mathrm{m}^{2}$ & $\begin{array}{c}43.31 \pm 12.07 \\
18-60\end{array}$ & $\begin{array}{c}33.05 \pm 9.43 \\
21.4-48\end{array}$ & $\mathrm{~T}=3.28$ & $0.002 *$ \\
\hline Metabolic syndrome $\mathrm{n} / \%$ & $6 / 24(25 \%)$ & $3 / 24(12.5 \%)$ & $X^{2}=1.23$ & 0.27 \\
\hline Hypertension $\mathrm{n} / \%$ & $10 / 24(41.6 \%)$ & $5 / 24(20.8 \%)$ & $X^{2}=2.43$ & 0.12 \\
\hline
\end{tabular}

Table 2:- Mean $\pm \mathrm{SD}$ (range) of the laboratory data of the studied subjects

\begin{tabular}{|c|c|c|c|c|}
\hline Variable & $\begin{array}{l}\text { Cases } \\
(n=24)\end{array}$ & $\begin{array}{c}\text { Control } \\
(\mathbf{n}=24)\end{array}$ & Test & $\mathbf{P}$ \\
\hline Osteopontin & $\begin{array}{c}187.08 \pm 141.1 \\
(95-600)\end{array}$ & $\begin{array}{c}107.42 \pm 35.8 \\
(960-180)\end{array}$ & 2.93 & $0.003^{*}$ \\
\hline FBG (mg/dl) & $\begin{array}{l}111.11 \pm 47.4 \\
(75-274.5)\end{array}$ & $\begin{array}{c}89.24 \pm 6.39 \\
(77.4-99)\end{array}$ & 1.32 & 0.19 \\
\hline Cholesterol(mg/dl) & $\begin{array}{c}248.88 \pm 62.89 \\
150-400\end{array}$ & $\begin{array}{l}174.42 \pm 34.87 \\
(111.2-239.8)\end{array}$ & 5.07 & $<0.001^{*}$ \\
\hline $\mathrm{TG}(\mathrm{mg} / \mathrm{dl})$ & $\begin{array}{c}230.75 \pm 64.6 \\
45.8-229\end{array}$ & $\begin{array}{c}102.74 \pm 50.76 \\
(32.9-233.7)\end{array}$ & 5.18 & $<0.001 *$ \\
\hline LDL-C(mg/dl) & $\begin{array}{c}116.79 \pm 30.35 \\
(58.5-169.8)\end{array}$ & $\begin{array}{c}99.4 \pm 33.39 \\
(33.4-174.6)\end{array}$ & 2.03 & $0.04 *$ \\
\hline HDL-C (mg/dl) & $\begin{array}{c}41.51 \pm 15.17 \\
(30-88.3)\end{array}$ & $\begin{array}{c}51.77 \pm 13.46 \\
(28-73.7)\end{array}$ & 3.1 & $0.002^{*}$ \\
\hline
\end{tabular}

HDL-C; high-density lipoprotein-cholesterol, TG ; triglycerides, LDL-C; low-density lipoprotein-cholesterol, , FBG; fasting blood glucose, $* \mathrm{P}<0.05$. 
Table 3:- Correlation between serum osteopontin level\&PASIscoreand patients data

\begin{tabular}{|l|c|c|c|c|}
\hline \multirow{2}{*}{ Variable } & Osteopontin & PASI & Score \\
\cline { 2 - 5 } & $\mathrm{r}$ & $\mathrm{P}$ & $\mathrm{r}$ & $\mathrm{P}$ \\
\hline Age & 0.36 & $\mathbf{0 . 0 3}$ & 0.03 & 0.90 \\
\hline BMI & -0.3 & 0.16 & 0.26 & $\mathbf{0 . 0 4}$ \\
\hline Duration of disease & -0.18 & 0.4 & 0.13 & 0.37 \\
\hline PASI score & 0.33 & 0.12 & - & - \\
\hline Systolic blood pressure & 0.39 & $\mathbf{0 . 0 2}$ & 0.28 & $\mathbf{0 . 0 4}$ \\
\hline Diastolic blood pressure & 0.41 & $\mathbf{0 . 0 1} *$ & 0.32 & $\mathbf{0 . 0 3}$ \\
\hline FBG & -0.29 & 0.18 & 0.39 & $\mathbf{0 . 0 2} *$ \\
\hline Cholesterol & 0.08 & 0.7 & 0.42 & $\mathbf{0 . 0 0 6} *$ \\
\hline TG & 0.09 & 0.68 & 0.40 & $\mathbf{0 . 0 1 *}$ \\
\hline LDL-C & -0.04 & 0.87 & 0.25 & $\mathbf{0 . 0 4} *$ \\
\hline HDL-C & -0.1 & 0.63 & -0.29 & $\mathbf{0 . 0 3 *}$ \\
\hline
\end{tabular}

HDL-C; high-density lipoprotein-cholesterol, TG ; triglycerides, LDL-C; low-density lipoprotein-cholesterol, FBG; fasting blood glucose. $* \mathrm{P}<0.05$.

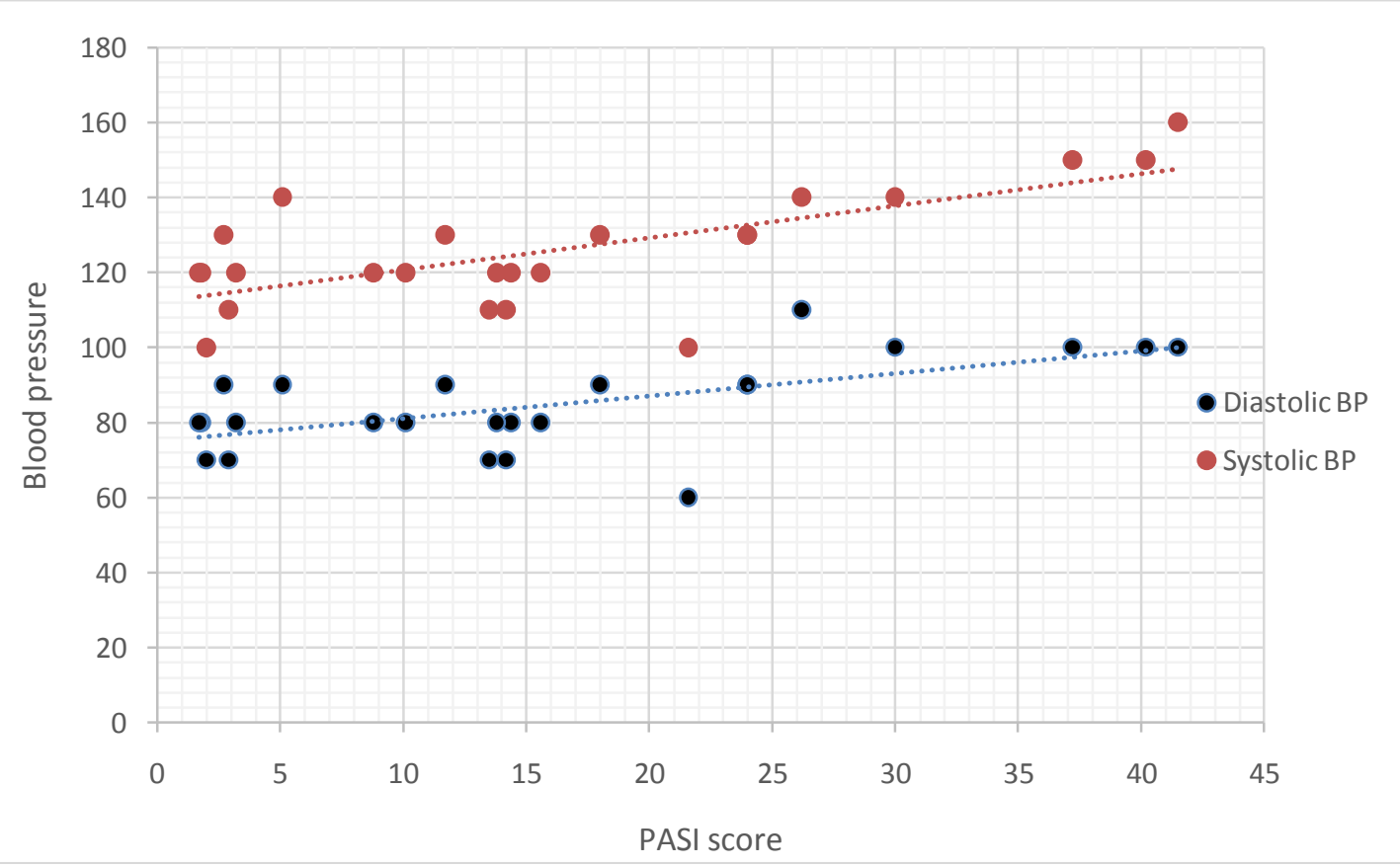

Fig.1a:-correlation between PASI score and blood pressure. 


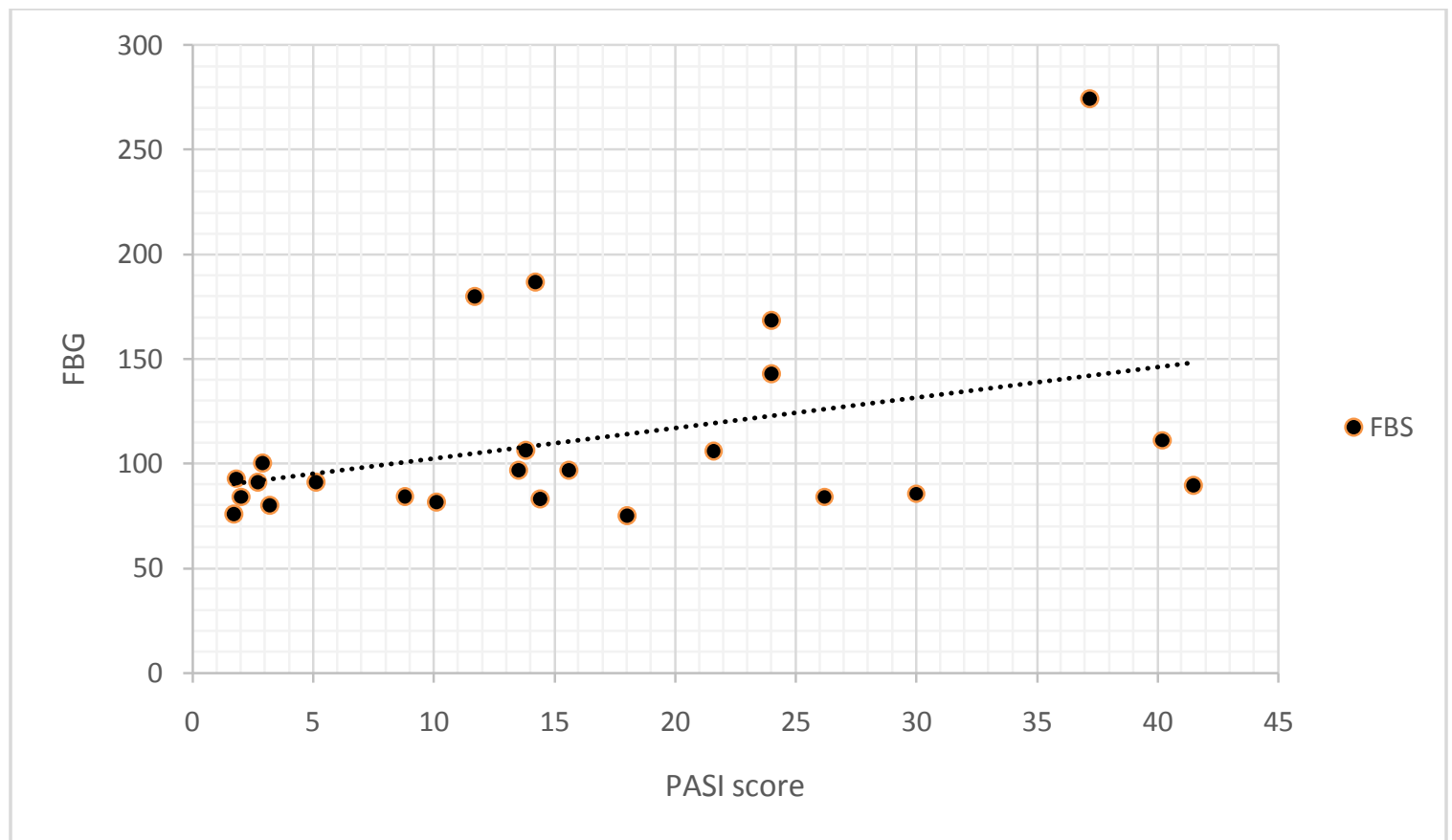

Fig1b:- correlation between PASI score and FBS .

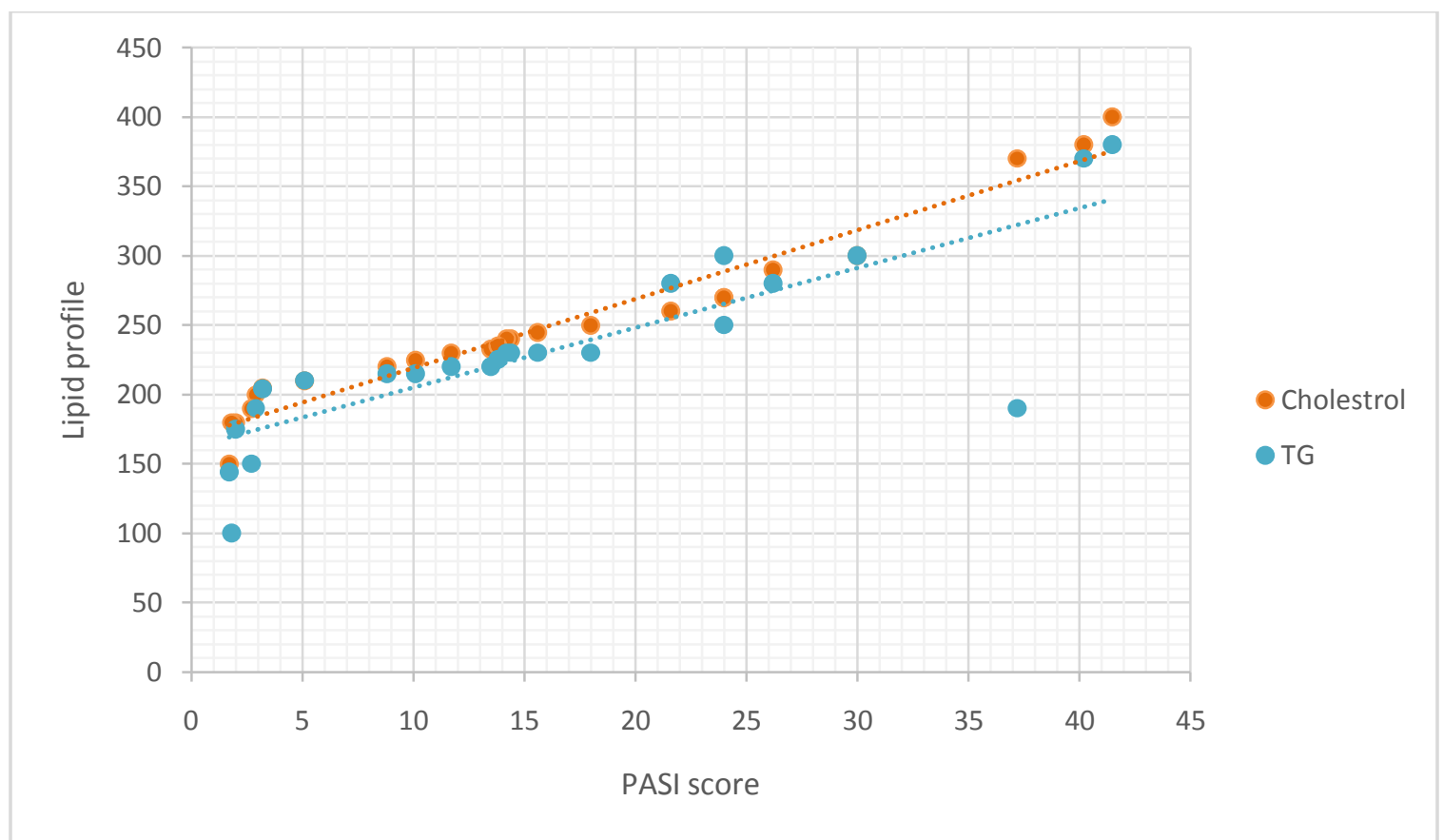

Fig 1c:- correlation between PASI score and lipid profile(cholesterol\&TG). 


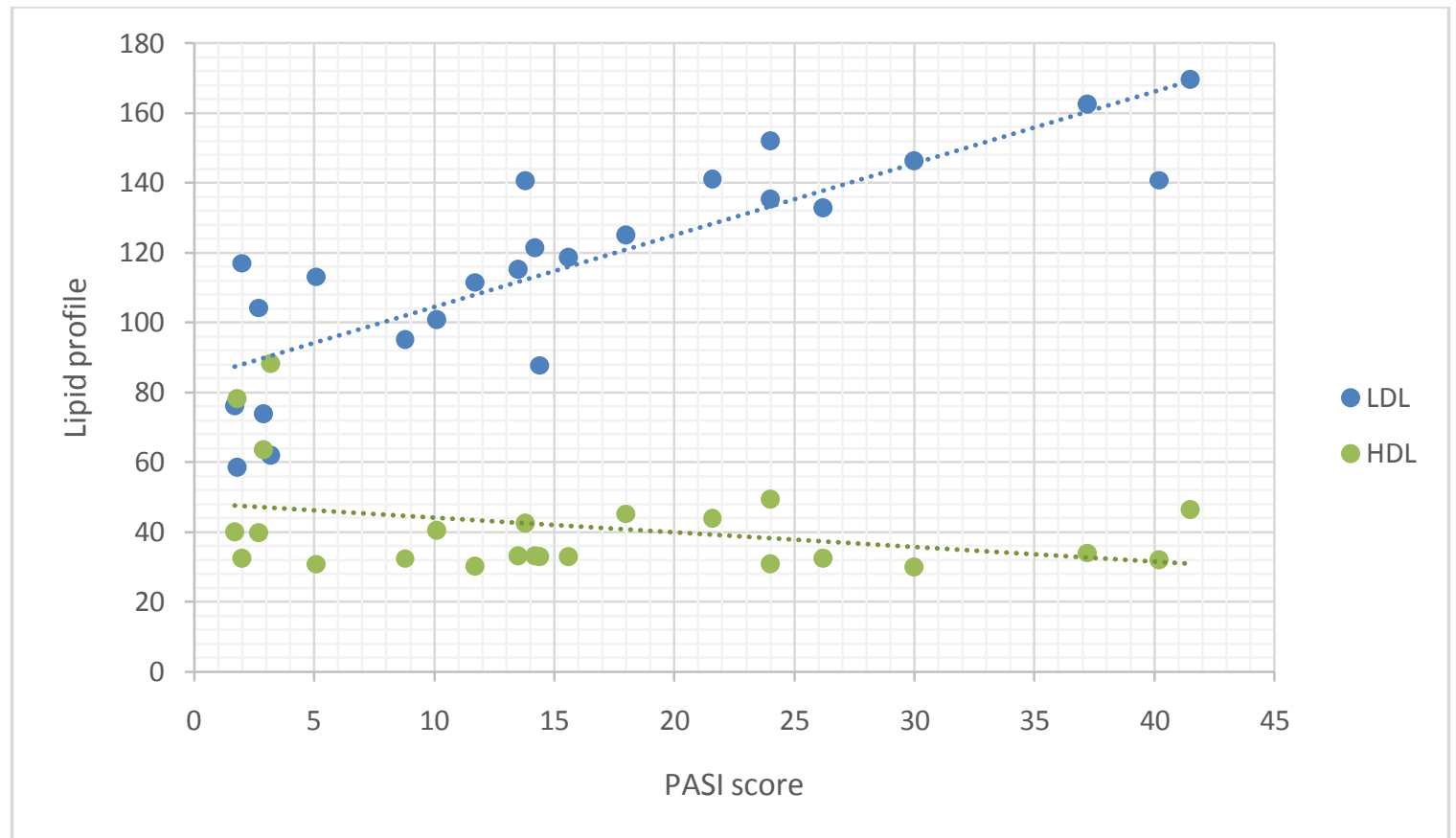

Fig.1d:- correlation between PASI score and lipid profile (LDL\&HDL).

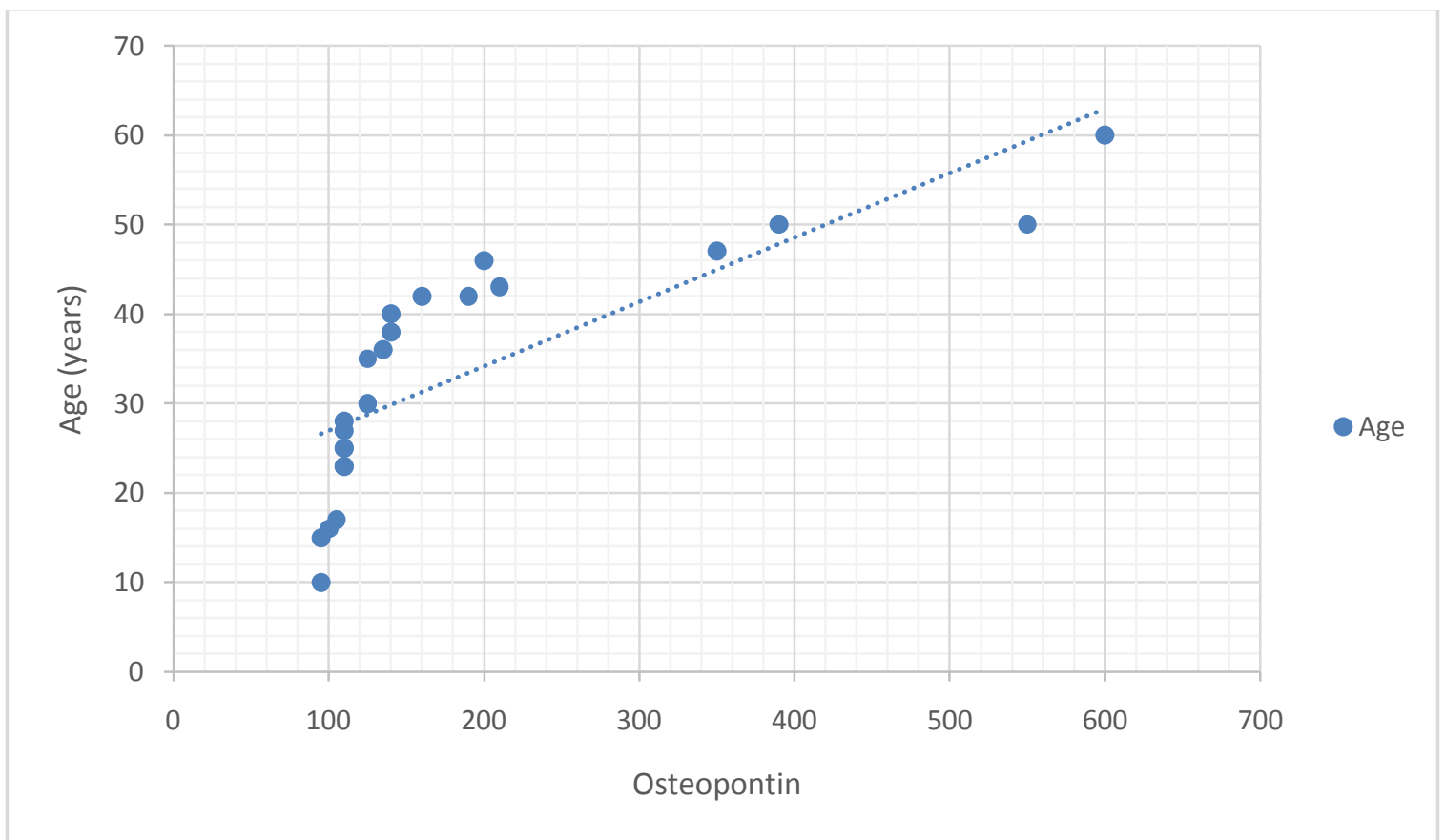

Fig 2a:- correlation between OPN level and age in cases group. 


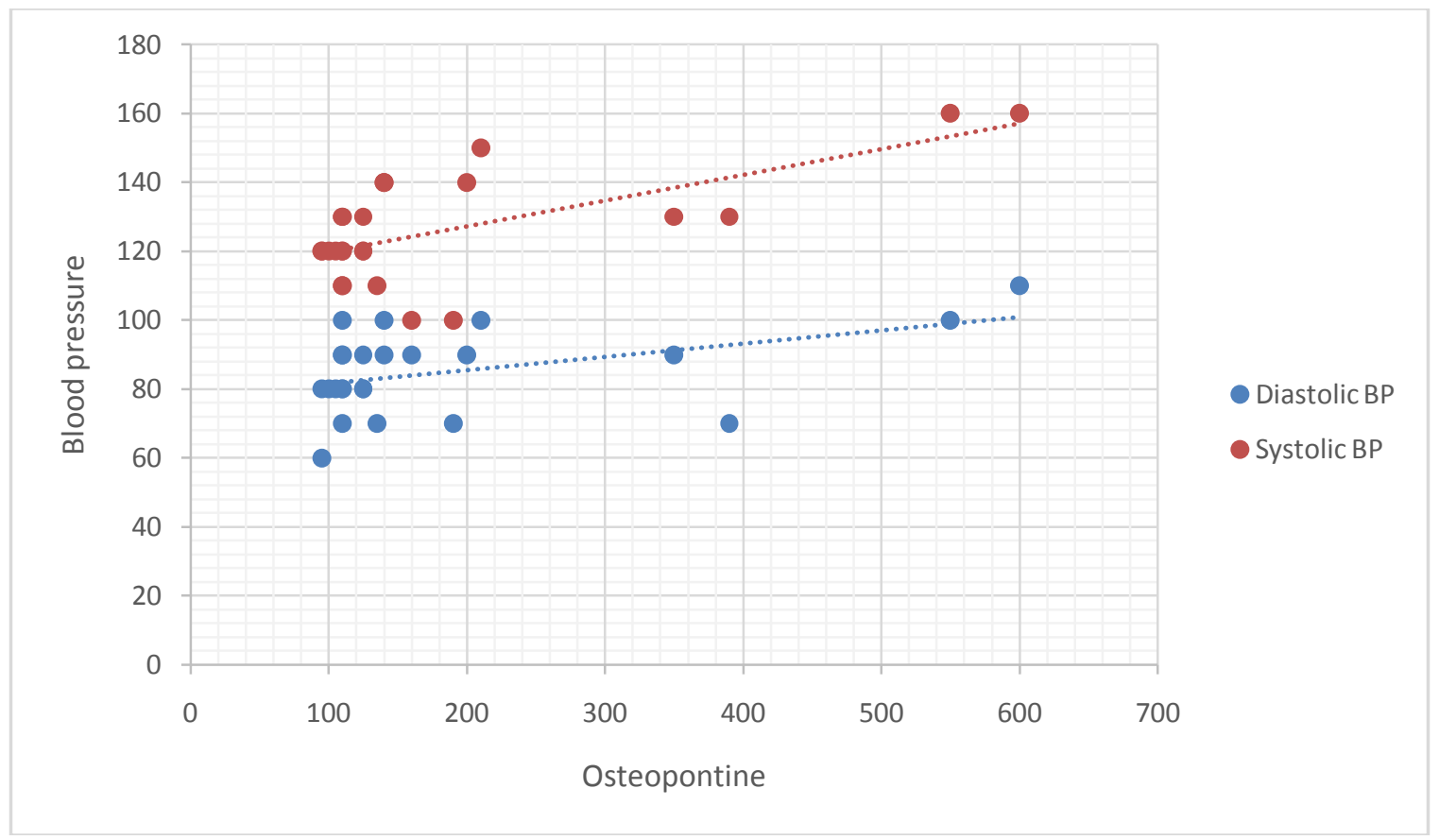

\section{Discussion:-}

Fig 2b:- correlationbetween serum OPN level and blood pressure.

Psoriasis is a chronic inflammatory immune-mediated disease affecting approximately 1-3\% of the general population. Psoriasis is not just a disease of skin and joints, but also is a systemic disease that is connected with a range of comorbidities especially metabolic syndrome and cardiovascular diseases(CVDs)[11].

This dermatosis is considered to be a T-cell mediated disease with active hyperproliferation of keratinocytes and abnormal vascular expansion within the superficial dermis. This expansion is mediated by angiogenesis, an active vaso-proliferative process which appears to be a key inflammatory response early in the pathogenesis of psoriasis [12].There is a number of Th lymphocyte subsets that have been detected in psoriatic lesions. Earlier reports indicated that activated Th1 cells in psoriatic lesions that produce IFN $\gamma$, TNF $\alpha$ and IL-2 were mainly involved in the pathogenesis of psoriasis [13].

Osteopontin is a phosphorylated acidic glycoprotein of pleiotropic properties and has been recently recognized as a potential inflammatory cytokine having a role in many diseases. OPN is produced by different cell lines and binds to several integrin receptors and is known to induce cell adhesion, migration, and survival of immune cells including neutrophils, macrophages, T cells, mast cells, and osteoclasts [14].Secreted OPN appears to have a role in the development of psoriasis through inhibiting keratinocyte apoptosis, thereby supporting enhanced epidermal proliferation. OPN promotes vessel formation subsequently supporting the influx of inflammatory cells. OPN has a pro-angiogenic effect on microvascular endothelial cells, and has been involved in the onset of angiogenesis through a mechanism mediated by IL-1[15].Moreover, there are reports suggest that OPN and MMP-9 may be regulated by $\mathrm{TNF} \alpha$, indicating that they may have a role in the pathogenesis of psoriasis [16].

Metabolic syndrome (Met.S) is a clinical entity comprising risk factors such as hypertension, glucose intolerance, hyperlipidemia, abdominal obesity and increased inflammatory state [17]. According to NCEP-ATP III (National Cholesterol Education Program Adult Treatment Panel III) metabolic syndrome involves 3 or more of the factors : Fasting plasma glucose $\geq 110$,Blood pressure: systolic $\geq 130$ \& diastolic $\geq 85$, Triglycerides $(\mathrm{mg} / \mathrm{dl}) \geq 150$,HDL-c $(\mathrm{mg}$ $/$ dl) : male $<40 \&$ female $<50$, Central obesity (waist circumference ) : male $>102 \mathrm{~cm} \&$ female $>88 \mathrm{~cm}$.

We aimed to assess metabolic syndrome and osteopontin and their associated systemic comorbidities in patients with psoriasis

In our study, FBS level was found to benonsignificantlymore in psoriatic cases rather than control. Cholesterol, LDL-c, TG levels weresignificantly elevated, andHDL-c levels were lowerin psoriatic cases than control. Also 
obesity was found to be statistically higher in psoriasis than control. Severity of psoriasis hadsignificant positive correlation with obesity,FBS, dyslipidemia and hypertension.

According to the results of our study, psoriasis is positively associated with metabolic syndrome, and patients with more severe psoriasis were exposed to have metabolic syndrome than those with milder psoriasis. This result is consistent with Armstrong et al.,2013[18] as they found that metabolic syndrome and its components was found to be more among subjects with psoriasis, and that patients with advanced psoriasis have greater odds of metabolic syndrome than those with milder psoriasis.

Psoriatic patients have a high prevalence of the Met.S. Psoriasis as a chronic inflammatory skin disorder is characterized by a variety of immunologic and inflammatory changes and may similarly predispose for those inflammatory disorders. This could likely be due to the effects of chronic inflammatory changes, in particular, the secretion of proinflammatory cytokines [19].

Chronic systemic inflammation induces endothelial dysfunction, altered glucose metabolism, and insulin resistance that play a significant role in the development of obesity, diabetes mellitus, dyslipidemia, and cardiovascular disease such as atherosclerosis and myocardial infarction or stroke [20]. On assessing the components of Met.S, psoriasis continues to stand as an independent risk factor with respect to altering levels of glucose tolerance and dyslipidemia. Subjects with psoriasis have a higher percentage of known DM. Patients with psoriasis have also an increased insulin resistance and probably worse impaired glucose tolerance, therefore a higher risk of diabetes[21].

Cytokines such as tumor necrosis factor (TNF)-alpha and interleukin-6 (IL-6) seem to play a central role. TNF- $\alpha$ plays a critical role in activation of innate and acquired immune responses leading to chronic inflammation, tissue damage and keratinocyte proliferation.TNF- $\alpha$ levels are markedly increased in skin lesions, synovium and serum of patients with psoriasis and these correlate with the severity of the disease. Decreased levels of TNF- $\alpha$ are associated with clinical resolution [22].

TNF- $\alpha$ may lead to insulin resistance by inhibiting insulin mediated tyrosine phosphorylation of the insulin receptor, as well as insulin receptor substrate-1. TNF- $\alpha$ has also been shown to be a potent activator of C-Jun amino-terminal kinase, which stimulates activator protein-1, a major regulator of pro-inflammatory activity and is connected with obesity[23]. Mouse models show that c-Jun amino-terminal kinase activity isabnormally elevated in obesity, while its absence is associated with decreased adiposity, improved insulin sensitivity and enhanced insulin receptor signaling[24].

We found that there was significant dyslipidemia in psoriatic cases than normal control. It was reported that macrophages activated by engulfing low density lipoprotein (LDL) immune complexes release large quantities ofTNF- $\alpha$. Treatment with TNF-inhibitors affect the increase of HDL levels, decreased LDL and triglyceride levels and strongly recommended the use of anti-TNF-amodalities, adalimumab and infliximab, for the treatment of obese psoriatics and suggested that anti-TNF- $\alpha$ treatment might prevent cardiovascular diseases in psoriasis [25].

Furthermore, interleukin-6, IL-8, Interferon $-\gamma$, IL1, and IL-17 arealso implicated in the generation of proatheromatous abnormalities like dyslipidemia, insulin resistance, endothelial dysfunction,clotting system activation, and pro-oxidative stress. TNF- $\alpha$ may affect endothelium dysfunction by decreasing the levels of nitric oxide synthase and cyclooxygenase-1[26].

C-reactive proteins [27]and cellular oxidativestress [28] may also be responsible for altered lipidmetabolism.Antipsoriatic drugs such as oral retinoids and cyclosporine, can bealso responsible for lipid profile disturbances in psoriatic patients because of their action on the circulating lipids. Including hypercholesterolemia,hypertriglyceridemia and low HDL-cholesterol[29].

Hypertriglyceridemia secondary to VLDL elevation is associatedwith both procoagulant and prothrombotic factors in the blood and affects the adhesiveness of platelets.Resting platelets circulate freely, adhering neither to each other nor to other cells. However, activated platelets adhere to all lipoproteins, especially VLDL. VLDL mediated platelet adhesion may play an important role in the progression of atherosclerosis. Furthermore, VLDL remnants are susceptible to retention within the arterial intima and thereby promoting atherosclerotic plaque growth [30, 31]. 
Regarding OPN, our study foundsignificantly elevated serum OPN in psoriatic patients than in healthy controls. AlsoRobati et al.,2016[31] found higher levels of serum osteopontin in psoriatic patients than in healthy controls. That elevated serum levels of osteopontinin our study were positively correlated with increasing age. Cheng et al.,2008[6] noticedthat, the level of OPN increased with increase of age.In our study plasma osteopontin values were not associated with sex, severity of psoriasis,duration of the disease or dyslipidemia Cheng et al.,2008[6] reported the same result that, plasma OPN levels were not associated with sex ,duration of the disease, disease severity or dyslipidemia.

Tossi et al.,2015,[32] measured serum levels of selenium ,prolactin and osteopontin in both psoriasis and healthy control groups, there was no significant difference in selenium and prolactin levels between the two groups, but OPN was significantly high psoriasis group.Lesional skin of psoriatic patients showed significant elevated OPN levels in comparison to controls [33, 34].In psoriasis, OPN secreted by keratinocytes inhibits keratinocyte apoptosis, thereby supporting enhanced epidermal proliferation, OPN acts as a pro-inflammatory agent that participates in the up-regulation of Th cell lineages, among which are the Th1 and Th17 cells. OPN secreted by keratinocytes, possibly stimulated through IFN $\gamma$ from T-cells and OPN from effector T cells attract additional inflammatory cells[15].

The relationship between the inflammatory cells present in psoriasis and OPN expression might be one of the responsible factors, where the environment is rich in inflammatory cells, such as macrophages, natural killer cells and $\mathrm{T}$ lymphocytes, which are known to be among the cells responsible for the expression of OPN [35].

Osteopontin is involved in the onset of angiogenesis which is considered as a corner stone in the psoriatic story. OPN expressed in inflammatory cells and endothelial cells of psoriatic patients .Its pro-angiogenic effect on microvascular endothelial cell is through promoting vessel formation subsequently supporting the influx of inflammatory cells [36].

The matrix metalloproteinase (MMP)-9 induced by OPN and TNF $\alpha$ acts as an angiogenesis promoting factor [37]. One study had demonstrated that OPN expression seems to be related to the CD34 expression, angiogenesis marker, expressed in the endothelial cell of psoriaticlesional skin[33].

In our study,elevated plasma OPN levels were found to be significantly associated with hypertension, but non significantly associated with fasting blood glucose.In agreement with us,cheng et al.,2008[6] found that elevated OPN levels were significantly associated with hypertension butnon significantly positively correlated with DM in psoriatic patients. Another study Robati et al.,2016[31]found higher levels of plasma OPN and mean intima-media wall thickness of the common carotid artery in psoriatic patients than healthy control and this support increased cardiovascular risk in psoriatic patients and the possible role of OPN .

OPN was found to be expressed in smooth muscle cells in the atherosclerotic lesion in angiogenicendothelial cell and macrophages. OPN modulates proliferation, migration and accumulation of smooth muscle cells and endothelial cell involved in repair and remodeling processes of the vasculature. Osteopontin expression increases under a variety of conditions of the heart diseases and is associated with increased myocyte apoptosis and myocardial dysfunction [7].Plasma OPN levels are higher in patients with coronary artery disease and have been correlated with its severity. Furthermore, plasma OPN has been reported to be a potential clinical marker for the prediction of atherosclerosis and further adverse cardiac events in patients with essential hypertension and patients with chronic stable angina[38].

In our results, we found non significantpositive correlations between plasma OPN and severity of psoriasis. On the contrary,El-Eishi et al., 2012[35]found positive correlation between plasma OPN and psoriasisseverity and found that plasma OPN was associated with the presence of metabolic syndrome components .

\section{Conclusion:-}

Psoriatic patients have a high prevalence of the metabolic syndrome.OPN involvement in psoriasis enlarges the list of cytokines able to stimulate the inflammatory response in this disease. OPN is involved in the pathophysiology of psoriasis, in the onset and worsening of psoriasis and lastly a possible association with disease severity. High level of OPN is correlated also with cardiovascular risks in psoriatic patients. The possibility of using this inflammatory cytokine as a new target in the treatment of psoriasis, anti-OPN antibodies, may eventually become a useful therapeutic 
approach in psoriasis.OPN may be used as early detector of cardiovascular problems in psoriatic patients and as a new target in decreasing cardiovascular risks in those patients.

\section{References:-}

1. Mahajan R and Handa S (2013) : Pathophysiology of psoriasis. Indian. J DermatolVenereolLeprol ; 79:1-9.

2. Abdelnoor AM and Al-Akl N (2013): Factors Involved in the Pathogenesis of psoriasis. Adv S Med Scien ;1(2): 7594.

3. VM Voiculescu, M Lupu, L Papagheorghe,C Giurcaneanu, and E Micu(2014):Psoriasis and Metabolic Syndrome scientific evidence and therapeutic implications,J Med Life. 2014 Oct-Dec; 7(4): 468-471.

4. Bassyouni IH, Bassyouni RH, Ibrahim NH, et al (2012) :Elevated Serum Osteopontin Levels in Chronic Hepatitis C Virus Infection:Association with Autoimmune Rheumatologic Manifestations. J ClinImmunol ; 32:1262-1269.

5. Sase S P, Nagane N., Ganu J V. Osteopontin: A Novel Protein Molecule. Indian Medical Gazette. 2012 Feb ; 145 (2): 62-66.

6. Cheng X, Yu X, Ding YJ, et al (2008) : The Th17/Treg imbalance in patients with acute coronary syndrome. ClinImmunol2008; 127: 89-97.

7. Mazzone A, Parri MS, Giannessi D, et al (2011): Osteopontin plasma levels and accelerated atherosclerosis in patients with CAD undergoing PCI: a prospective clinical study. J Coron Artery Dis ; 22(3):179-187.

8. Alain-P G.,Hervé C.,DanièleD.,Mark K et al (2001):Time Course of Osteopontin, Osteocalcin, and Osteonectin Accumulation and Calcification After Acute Vessel Wall Injury, J HistochemCytochem January 2001 vol. 49 no. $179-86$

9. Mattei PL1, Corey KC, Kimball AB.(2013):Psoriasis Area Severity Index (PASI) and the Dermatology Life Quality Index (DLQI): the correlation between disease severity and psychological burden in patients treated with biological therapies.J EurAcadDermatolVenereol. 2014 Mar;28(3):333-7. . Epub 2013 Feb 21.

10. Langley RG and Ellis CN (2004) : Evaluating psoriasis with Psoriasis area and Severity Index, Psoriasis Global Assessment and Lattice System Physician Global Assessment. J Am AcadDermatol ; 51:563-569.

11. Kılıç A and Cakmak S (2013) :Psoriasis and comorbidities. EMJ Dermatol ; 1:78-85.

12. Lima H, Dogan S, Atakan N, et al (2013): Psoriasis, types, causes and medications. InTech ; 51(1):978-953.

13. Lowes MA,Suárez-Fariñas M and Krueger JG (2014) : Immunology of Psoriasis. J Annu Rev Immunol; 32:227-255.

14. Kahles F, Findeisen H M and Bruemme D (2014): Osteopontin: A novel regulator at the cross roads of inflammation, obesity and diabetes .J MolMetab ; 3(6):384-393.

15. Buback F, Renkl AC, Schulz G, et al (2009): Osteopontin and the skin: multiple emerging roles in cutaneous biology and pathology. J ExpDermatol ; 18:750-759.

16. Buommino E, Filippis A, Gaudiello F, et al (2012): Modification of osteopontin and MMP-9 levels in patients with psoriasis on anti-TNF $\alpha$ therapy. Arch Dermatol Res; 304(6):481-5.

17. Balta I, Balta S, Demirkol S, et al (2013): Other inflammatory markers and related factors should be kept in mind in metabolic syndrome with psoriasis patients. Arch DermatolRes ; 305:459-460 .

18. Armstrong AW, Harskamp CT, Armstrong EJ (2013): Psoriasis and metabolic syndrome: a systematic review and meta-analysis of observational studies. J Am AcadDermatol ; 68:654-662.

19. Nakajima H, Nakajima K, Tarutani M, Sano S (2012) The role of pigment epithelium-derived factor as an adipokine in psoriasis. Arch Dermatol Res 304(1):81-84

20. Boehncke W., Boehncke S., Tobin A., Kirby B. (2011) The 'psoriatic march': a concept of how severe psoriasis may drive cardiovascular comorbidity. ExpDermatol 20: 303-307

21. Albareda M, Ravella A, Castelló M et al (2014):Metabolic syndrome and its components in patients with psoriasis.Springer plus;3:612.

22. Joshi R. (2004):Immunopathogenesis of psoriasis. Indian J DermatolVenereolLeprol; 70:10-2.

23. Farshichian M, Zamanian A, Farshichian M, et al (2007) :Serum lipid levels in Iranian patients withpsoriasis. J EurAssocDermatolVenereol; 21: 802-805.

24. JacobD.,Dahlia W.,BaytaD.,JonathanS.,et al (2008):Ssoriasis and Dyslipidaemia:APopulatio - based study,ActaDermVenereol 2008;88:561-565

25. Strober BE, Poulin Y, Kerdel FA, et al (2011): Switching to adalimumab for psoriasis patients with a suboptimal response to etanercept, methotrexate, or phototherapy: efficacy and safety results from an open-label study. J Am AcadDermatol; 664:671.

26. Karadag AS, Yavuz B, Ertugrul DT, et al (2010): Is psoriasis a preatherosclerotic disease? Increased insulin resistance and impaired endothelial function in patients with psoriasis. Int J Dermatol;48(6):642-646.

27. Escarcega RO, Garcia-Carrasco M, Fuentes-Alexandro S, et al (2006): Insulin resistance, chronic inflammatory state and the link with systemic lupus erythematosus-related coronary disease. Autoimmune Rev; 6:48-53. 
28. Rocha-Preira P, Santos-Silva A, Rebelo I, et al (2001): Dyslipdemia and oxidative stress inmild and in severe psoriasis as a risk for cardiovascular disease.ClinChimActa; 303:33-90.

29. WakkeeM,ThioHB,Prens EP, et al(2007): Unfavorable cardiovascular risk profiles in untreated andtreated psoriasis patients. Atherosclerosis; 190: 1-9.

30. Lotus Mallbris, MD, PhD ,FredrikGranath, Anders Hamsten,ET AL (2006):Psoriasis is associated with lipid abnormalities at the onset of skin disease,JAAD, April 2006Volume 54, Issue 4, Pages 614-621

31. Robati RM1, Partovi-Kia M1, Sadat-Amini H1, Haghighatkhah HR2, et al (2016): Serum osteopontin level and common carotid artery intima-media wall thickness in psoriasis. Int J Dermatol. 2016 May;55(5):e262-7. Epub 2016 Jan 15.

32. Toossi P1, Sadat Amini SH1, Sadat Amini MS2, Partovi Kia M1, et al(2015):Assessment of serum levels of osteopontin, selenium and prolactin in patients with psoriasis compared with healthy controls, and their association with psoriasis severity.ClinExpDermatol. 2015 Oct;40(7):741-6. doi: 10.1111/ced.12657. Epub 2015 May 19.

33. Amin MM and Azim ZA (2012): Immunohistochemical study of osteopontin, Ki-67 and CD34 of psoriasis in Mansoura, Egypt. Indian J PatholMicrobiol ; 55(1):56-60.

34. El-Eishi NH, Kadry D, Hegazy RA, Rashed L (2012) : Estimation of tissue osteopontin levels before and after different traditional therapeutic modalities in psoriatic patients. J EurAcadDermatol Venereol;27(3):351-5.

35. Gaspari AA (2006): Innate and adaptive immunity and the pathophy-siology of psoriasis .J Am AcadDermatol ; 54(3suppl2):67-80.

36. Giachelli CM and Steitz (2000) :S.Osteopontin: A Versatile regulator of inflammation and biomineralization. J Matrix Biol ; 19(7):615-622.

37. Nissinen L and Kähäri VM (2014): Matrix metalloproteinases in inflammation . J BiochimBiophysActa; 1840(8):2571-2580.

38. Abdel-Azeez HA and Al-Zaky M (2010): Plasma osteopontin as a predictor of coronary artery disease: association with echocardiographic characteristics of atherosclerosis. J Clin Lab Anal ;24(3):201-206. 